\title{
Dependence of MIMO System Performance on the Joint Properties of Angular Power
}

\author{
Terence Betlehem ${ }^{\dagger}$, Tharaka A. Lamahewa ${ }^{\dagger}$ \\ and Thushara D. Abhayapala ${ }^{\dagger \ddagger}$ \\ $\dagger$ Research School of Information Science and Engineering, Australian National University, Australia \\ † Wireless Signal Processing Program, National ICT Australia Ltd. \\ Canberra, Australia \\ [terence.betlehem, tharaka.lamahewa, thushara.abhayapala] @anu.edu.au
}

\begin{abstract}
In this paper, we use a novel MIMO channel model to characterize the dependence of ergodic capacity and diversity order on the joint statistics of the angular power density. The scattering environment of a MIMO channel is characterized by a double directional angular power distribution, describing the power transferred in each direction from transmitter aperture to receiver aperture. Angular power, which is typically separable Kronecker-modelled, is here generalized to include joint distribution properties using well-known bivariate probability density functions. We show that the joint properties of the power density, namely the shape and the orientation of power distribution contours, have significant impact on capacity and diversity of non-line-of-sight (NLOS) channels.
\end{abstract}

\section{INTRODUCTION}

With recent development of practical MIMO systems, there is need to quantify the attainable MIMO performance over realistic channels. Many options for channel models are now available but either require ray-tracing, non-linear parametrizations, or restrict simulation to fixed array geometries at the transmitter and receiver. We present a model for arbitrary array geometries, and use it to characterize the effect of nonKronecker channel properties on MIMO system performance, as a function of the angular power distribution.

A rich body of literature exists for the characterization of MIMO channels. For non-line-of-sight channels the channel gains are dominated by their second order statistics [1]. To model the second order channel statistics, authors commonly use geometric models based on parametrizations of the double directional power distribution [2]. However, many authors still use the oversimplified Kronecker model, where signal correlation at the transmitter and receiver are assumed independent. This model poorly estimates the capacity [3] and antenna correlations [4] in many cases.

In this paper, we study the joint power distribution parameters on the performance of a MIMO system. We use a novel model for non-line-of-sight channels that allows an antenna geometry-free study of the channel. We quantify the effect on ergodic capacity and diversity order of (i) the level of correlation between transmit and receive power spectra and (ii) the orientation of distribution contours in the transmitangle/receive-angle plane.

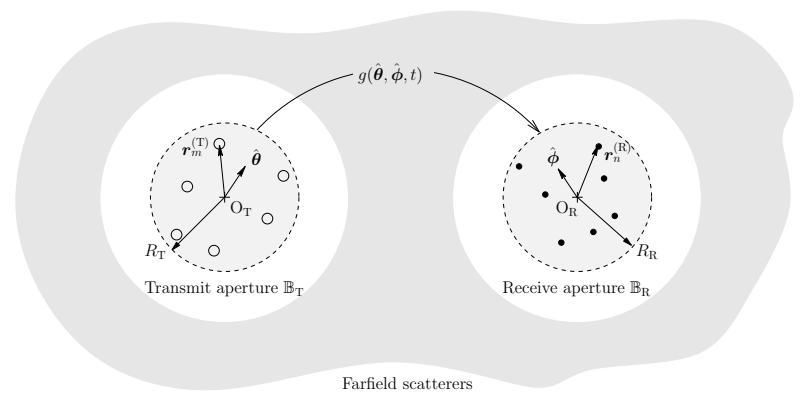

Fig. 1. The channel model. Scattering is modelled with the scattering gain $g(\theta, \phi, t)$. Each transmitter (o) is positioned at $\boldsymbol{r}_{m}^{(\mathrm{T})}$ within circular aperture of radii $R_{\mathrm{T}}$ and receiver (.) at $\boldsymbol{r}_{n}^{(\mathrm{R})}$ within aperture of radius $R_{\mathrm{R}}$.

\section{Channel Model}

MIMO system performance is controlled by the correlation between the signals at the transmit and receive antennas. In this section, we model the channel correlation. We present a 2D model, which is reasonable for urban channel environments.

Consider transmission of data from $n_{\mathrm{R}}$ transmitter antennas over a flat fading channel to $n_{\mathrm{R}}$ receiver antennas. Let $\boldsymbol{r}_{m}^{(\mathrm{T})}$ be the $m$ th transmitter antenna position with respect to an transmitter origin $\mathrm{O}_{\mathrm{T}}$ and $\boldsymbol{r}_{n}^{(\mathrm{R})}$ be the $n$th receiver antenna position with respect to a receiver origin $\mathrm{O}_{R}$ (as shown in Fig. 1). Transmitter and receiver antennas lie within finite circular apertures of radius $R_{\mathrm{T}}$ and $R_{\mathrm{R}}$ respectively. Transmitters transmits the $n_{\mathrm{T}} \times 1$ vector signal $\boldsymbol{x}(t)$ over the time-varying channel described by matrix $\boldsymbol{H}(t)$ to receive the $n_{\mathrm{R}} \times 1$ vector signal $\boldsymbol{y}(t)$ at receiver antennas. Matrix $\boldsymbol{H}(t)$ houses the transfer functions $[\boldsymbol{H}]_{n m} \triangleq h_{n m}(t)$ from each transmitter antenna $m$ to receiver antenna $n$. Defining the $n_{\mathrm{R}} \times 1$ vector of additive white Gaussian noise $\boldsymbol{n}(t)$ at the receivers, we write:

$$
\boldsymbol{y}(t)=\boldsymbol{H}(t) \boldsymbol{x}(t)+\boldsymbol{n}(t) .
$$

Assume that all scatterers lie in the far-field. The scattering environment causes transmitter signals to propagate in as plane waves with a different amplitude for each direction. Define the scattering gain $g(\theta, \phi, t)$ as the complex gain at time $t$ of the signal propagating out from the transmitter origin in direction $\theta$ and arrives at the receiver origin in direction $\phi$. We can then 
write:

$$
h_{n m}(t)=\int_{2 \pi} \int_{2 \pi} g(\theta, \phi, t) e^{i k\left(\boldsymbol{x}_{m} \cdot \hat{\boldsymbol{\theta}}-\boldsymbol{y}_{n} \cdot \hat{\boldsymbol{\phi}}\right)} d \phi,
$$

where $\hat{\boldsymbol{\theta}}$ and $\hat{\boldsymbol{\phi}}$ are unit vectors of polar coordinates $(1, \phi)$ and $(1, \theta)$ and $k$ is the wave number. Assume slow fading so that the channel remains static over the symbol time. Then $h_{n m}$ and $g$ are not dependent on $t$ over each symbol.

To investigate the statistics of non-line-of-sight scattering, $g(\theta, \phi)$ is assumed to be a zero mean Gaussian random variable at each angle pair $(\theta, \phi)$. Assume $g(\theta, \phi)$ is uncorrelated between different angles:

$$
E_{g}\left\{g(\theta, \phi) g^{*}(\vartheta, \varphi)\right\}=\mathcal{P}(\theta, \phi) \delta_{\theta \vartheta} \delta_{\phi \varphi},
$$

where $\delta_{\phi \varphi}$ is the Kronecker delta function and $*^{*}$ is the complex conjugate and $\mathcal{P}(\theta, \phi) \triangleq E_{g}\left\{|g(\theta, \phi)|^{2}\right\}$ is the power density of scatterers, interpreted as the average energy transmitted in direction $\theta$ and received in direction $\phi .^{1}$ The expectation is computed over all possible realizations of the scattering environment $g(\theta, \phi)$. Equation 3 is referred to as the wide-sense stationary uncorrelated scatterer (WSSUS) assumption [5].

Without loss of generality, we normalize the average energy of each channel coefficient to 1 , which is equivalent to normalizing $\mathcal{P}(\theta, \phi)$ :

$$
\int_{2 \pi} \int_{2 \pi} \mathcal{P}(\theta, \phi) d \theta d \phi=1 .
$$

Statistics of $g(\theta, \phi)$ and $\boldsymbol{H}$ are dependent entirely upon $\mathcal{P}(\theta, \phi)$.

The univariate marginals of the angular power are understood as the power density of scatterers at the transmitter:

$$
\mathcal{P}_{\mathrm{T}}(\theta) \triangleq \int_{2 \pi} \mathcal{P}(\theta, \phi) d \phi,
$$

and power density of scatterers at the receiver:

$$
\mathcal{P}_{\mathrm{R}}(\phi) \triangleq \int_{2 \pi} \mathcal{P}(\theta, \phi) d \theta
$$

Suitable functional forms for the univariate scatterer distributions have been well-studied [6]. These distributions characterize the properties of the separable Kronecker model, where it can be shown that $\mathcal{P}(\theta, \phi)=\mathcal{P}_{\mathrm{T}}(\theta) \mathcal{P}_{\mathrm{R}}(\phi)$ [4]. This paper relaxes the Kronecker assumption, by additionally describing power density with a jointness parameter.

The MIMO model is used to calculate the second order statistics $E_{g}\left\{h_{n m} h_{n^{\prime} m^{\prime}}^{*}\right\}$ of the entries of $\boldsymbol{H}$. These statistics can be collected in the channel correlation matrix $\boldsymbol{R}_{\boldsymbol{H}} \triangleq$ $E_{g}\left\{\overrightarrow{\boldsymbol{H}} \overrightarrow{\boldsymbol{H}}^{\dagger}\right\}$ where vectorize operator $\vec{\bullet}$ stacks the columns of a matrix and.$^{\dagger}$ is the Hermitian operator.

\section{Fourier ANALysis of ChANNEl MATRIX}

We now derive a compact expression for $\boldsymbol{R}_{\boldsymbol{H}}$. By transforming functions into the Fourier domain, we decompose the channel matrices $\boldsymbol{H}$ and $\boldsymbol{R}_{\boldsymbol{H}}$ into components depending separately on the antenna geometry and scattering environment.

\footnotetext{
${ }^{1}$ Power density of scatterers is not to be confused with the probability density function of the scattering gain, which we model as complex Gaussian.
}

\section{A. Fourier Decomposition}

Perform the double Fourier expansion of $\mathcal{P}(\theta, \phi)$,

$$
\begin{gathered}
\mathcal{P}(\theta, \phi)=\frac{1}{(2 \pi)^{2}} \sum_{\ell=-\infty}^{\infty} \sum_{\ell^{\prime}=-\infty}^{\infty} \gamma_{\ell \ell^{\prime}} e^{i\left(\ell \theta-\ell^{\prime} \phi\right)}, \\
\gamma_{\ell \ell^{\prime}}=\int_{2 \pi} \int_{2 \pi} \mathcal{P}(\theta, \phi) e^{-i\left(\ell \theta-\ell^{\prime} \phi\right)} d \theta d \phi,
\end{gathered}
$$

where $\gamma_{\ell \ell^{\prime}}$ is the Fourier coefficient of $\mathcal{P}(\theta, \phi)$. Since $\mathcal{P}(\theta, \phi) \in \mathbb{R}$, we know that $\gamma_{\ell\left(-\ell^{\prime}\right)}^{*}=\gamma_{(-\ell) \ell^{\prime}}$. Substituting (5a) into (4) we see the power density of scatterers around transmitter and receiver are a function of $\gamma_{\ell 0}$ and $\gamma_{0 \ell^{\prime}}$ :

$$
\begin{gathered}
\mathcal{P}_{\mathrm{T}}(\theta)=\frac{1}{2 \pi} \sum_{\ell=-\infty}^{\infty} \gamma_{\ell 0} e^{i \ell \theta}, \\
\mathcal{P}_{\mathrm{R}}(\phi)=\frac{1}{2 \pi} \sum_{\ell^{\prime}=-\infty}^{\infty} \gamma_{0 \ell^{\prime}} e^{-i \ell^{\prime} \phi} .
\end{gathered}
$$

Similarly write $g(\theta, \phi)$ as the double Fourier expansion:

$$
\begin{gathered}
g(\theta, \phi)=\frac{1}{(2 \pi)^{2}} \sum_{\ell=-\infty}^{\infty} \sum_{\ell^{\prime}=-\infty}^{\infty} \beta_{\ell \ell^{\prime}} e^{i\left(\ell \theta-\ell^{\prime} \phi\right)}, \\
\beta_{\ell \ell^{\prime}}=\int_{2 \pi} \int_{2 \pi} g(\theta, \phi) e^{-i\left(\ell \theta-\ell^{\prime} \phi\right)} d \theta d \phi
\end{gathered}
$$

where $\beta_{\ell \ell^{\prime}}$ is the Fourier coefficient of $g(\theta, \phi)$.

Due to the limited aperture sizes, (7a) can be truncated and from (2) each $h_{n m}(t)$ written as a finite sum of the $\beta_{\ell \ell^{\prime}}$ coefficients. Drawing from [7], the channel matrix $\boldsymbol{H}$ can be decomposed into three matrices:

$$
\boldsymbol{H}=\boldsymbol{J}_{\mathbf{R}} \boldsymbol{\beta} \boldsymbol{J}_{\mathbf{T}}^{\dagger}
$$

where

$$
\boldsymbol{\beta}=\left[\begin{array}{ccc}
\beta_{\left(-N_{\mathrm{R}}\right)\left(-N_{\mathrm{T}}\right)} & \ldots & \beta_{\left(-N_{\mathrm{R}}\right) N_{\mathrm{T}}} \\
\beta_{\left(-N_{\mathrm{R}}+1\right)\left(-N_{\mathrm{T}}\right)} & \cdots & \beta_{\left(-N_{\mathrm{R}}+1\right) N_{\mathrm{T}}} \\
\vdots & \ddots & \vdots \\
\beta_{N_{\mathrm{R}}\left(-N_{\mathrm{T}}\right)} & \cdots & \beta_{N_{\mathrm{R}} N_{\mathrm{T}}}
\end{array}\right],
$$

is a matrix which completely characterizes the effect of the scattering environment, $\boldsymbol{J}_{\mathbf{T}}$ and $\boldsymbol{J}_{\mathbf{R}}$ are matrices describing the effect of the transmitter and receiver antenna geometries

$$
\boldsymbol{J}_{\mathrm{S}}=\left[\begin{array}{ccc}
\mathcal{J}_{-N_{\mathrm{S}}}\left(\boldsymbol{r}_{1}^{(\mathrm{S})}\right) & \ldots & \mathcal{J}_{N_{\mathrm{S}}}\left(\boldsymbol{r}_{1}^{(\mathrm{S})}\right) \\
\vdots & \ddots & \vdots \\
\mathcal{J}_{-N_{\mathrm{S}}}\left(\boldsymbol{r}_{n_{\mathrm{S}}}^{(\mathrm{S})}\right) & \ldots & \mathcal{J}_{N_{\mathrm{S}}}\left(\boldsymbol{r}_{n_{\mathrm{S}}}^{(\mathrm{S})}\right)
\end{array}\right],
$$

where $\mathrm{S}=\mathrm{T}$ for $\boldsymbol{J}_{\mathbf{T}}$ and $\mathrm{S}=\mathrm{R}$ for $\boldsymbol{J}_{\mathbf{R}}$, and if vector $\boldsymbol{r}$ is defined in polar coordinates as $\left(r, \theta_{r}\right)$, then

$$
\mathcal{J}_{\ell}(\boldsymbol{r}) \triangleq(-i)^{\ell} J_{\ell}(k r) e^{i \ell \theta_{r}},
$$

$N_{\mathrm{T}}=\left\lceil e k R_{\mathrm{T}} / 2\right\rceil$ and $N_{\mathrm{R}}=\left\lceil e k R_{\mathrm{R}} / 2\right\rceil$ are the aperture dimensionalities of transmitter and receiver respectively and $J_{m}(\cdot)$ is the Bessel function of the first kind of order $m$.

We next model the scattering matrix $\boldsymbol{\beta}$ as a random matrix. 


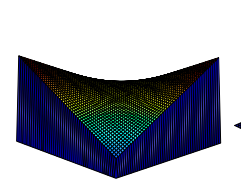

(a)

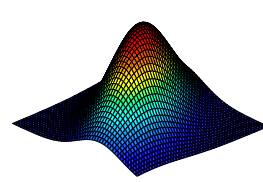

(b)

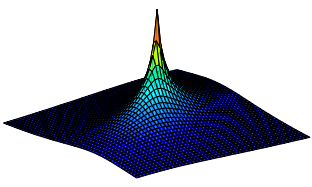

(c)
Fig. 2. Shapes of scatterer distributions used for joint distribution modelling: (a) Morgenstern (here $\rho_{m}=0.7$ ), (b) bivariate Gaussian $\left(\theta_{v}=\pi / 6, \sigma_{1}=2\right.$, $\left.\sigma_{2}=1\right)$ and (c) bivariate Laplacian $\left(\theta_{v}=\pi / 6, \sigma_{1}=4, \sigma_{2}=2\right)$.

\section{B. Statistics of Scattering Environment}

Since the scattering gain is zero mean Gaussian, the statistics of the elements of $\boldsymbol{\beta}$ are also governed by its correlation matrix $\boldsymbol{R}_{\boldsymbol{\beta}} \triangleq E_{g}\left\{\overrightarrow{\boldsymbol{\beta}} \overrightarrow{\boldsymbol{\beta}}^{\dagger}\right\}$. From (7b) each second order statistic $E_{g}\left\{\beta_{p p^{\prime}} \beta_{q q^{\prime}}^{*}\right\}$ can be calculated as:

$$
\begin{aligned}
E_{g}\left\{\beta_{p p^{\prime}} \beta_{q q^{\prime}}^{*}\right\}= & \int_{2 \pi} \int_{2 \pi} \int_{2 \pi} \int_{2 \pi} e^{-i\left(p \theta-p^{\prime} \phi\right)} e^{i\left(q \vartheta-q^{\prime} \varphi\right)} \\
& \times E_{g}\left\{g(\theta, \phi) g^{*}(\vartheta, \varphi)\right\} d \theta d \phi d \vartheta d \varphi
\end{aligned}
$$

Applying the WSSUS property (3):

$$
E_{g}\left\{\beta_{p p^{\prime}} \beta_{q q^{\prime}}^{*}\right\}=\int_{2 \pi} \int_{2 \pi} \mathcal{P}(\theta, \phi) e^{-i(p-q) \theta} e^{i\left(p^{\prime}-q^{\prime}\right) \phi} d \theta d \phi .
$$

We see by comparison with (5b) that $E_{g}\left\{\beta_{p p^{\prime}} \beta_{q q^{\prime}}^{*}\right\}=$ $\gamma_{(p-q)\left(p^{\prime}-q^{\prime}\right)}$ from which a block Toeplitz structure can be inferred for $\boldsymbol{R}_{\beta}$ :

$$
\boldsymbol{R}_{\boldsymbol{\beta}}=\left[\begin{array}{cccc}
\boldsymbol{\Gamma}_{0} & \boldsymbol{\Gamma}_{-1} & \ldots & \boldsymbol{\Gamma}_{-2 N_{\mathrm{T}}} \\
\boldsymbol{\Gamma}_{1} & \boldsymbol{\Gamma}_{0} & \ldots & \boldsymbol{\Gamma}_{-2 N_{\mathrm{T}}+1} \\
\vdots & \vdots & \ddots & \vdots \\
\boldsymbol{\Gamma}_{2 N_{\mathrm{T}}} & \boldsymbol{\Gamma}_{2 N_{\mathrm{T}}-1} & \ldots & \boldsymbol{\Gamma}_{0}
\end{array}\right]
$$

where $\boldsymbol{\Gamma}_{\ell}$ is Toeplitz:

$$
\boldsymbol{\Gamma}_{\ell}=\left[\begin{array}{cccc}
\gamma_{0 \ell} & \gamma_{(-1) \ell} & \ldots & \gamma_{\left(-2 N_{\mathrm{R}}\right) \ell} \\
\gamma_{1 \ell} & \gamma_{0 \ell} & \ldots & \gamma_{\left(-2 N_{\mathrm{R}}+1\right) \ell} \\
\vdots & \vdots & \ddots & \vdots \\
\gamma_{2 N_{\mathrm{R}} \ell} & \gamma_{\left(2 N_{\mathrm{R}}-1\right) \ell} & \ldots & \gamma_{0 \ell}
\end{array}\right]
$$

By virtue of the $\gamma_{\ell\left(-\ell^{\prime}\right)}=\gamma_{(-\ell) \ell^{\prime}}^{*}$ property, $\boldsymbol{\Gamma}_{-\ell}=\boldsymbol{\Gamma}_{\ell}^{\dagger}$.

The statistics of $\boldsymbol{H}$ are governed by covariance $\boldsymbol{R}_{\boldsymbol{\beta}}$ of $\boldsymbol{\beta}$ and the antenna geometry. From (8) and Kronecker relation $\overrightarrow{\boldsymbol{A B C}}=\left(\boldsymbol{A}^{T} \otimes \boldsymbol{C}\right) \overrightarrow{\boldsymbol{B}}$ where $\otimes$ is the Kronecker product and .$^{T}$ is the vector transpose operator [8], the channel correlation matrix $\boldsymbol{R}_{\boldsymbol{H}}$ is related to $\boldsymbol{R}_{\boldsymbol{\beta}}$ through

$$
\boldsymbol{R}_{\boldsymbol{H}}=\left(\boldsymbol{J}_{\mathbf{T}}^{*} \otimes \boldsymbol{J}_{\mathbf{R}}\right) \boldsymbol{R}_{\boldsymbol{\beta}}\left(\boldsymbol{J}_{\mathbf{T}}^{*} \otimes \boldsymbol{J}_{\mathbf{R}}\right)^{\dagger}
$$

This equation separates antenna correlation into the antenna geometry term $\boldsymbol{J}_{\mathbf{T}}{ }^{*} \otimes \boldsymbol{J}_{\mathbf{R}}$ and scattering environment correlation term $\boldsymbol{R}_{\boldsymbol{\beta}}$. We can then investigate separately the contributions of either component to MIMO performance. Equation 13 describes both Kronecker and non-Kronecker channels.

\section{Bi-Angular Power Distributions}

In this paper we consider the dependence on joint properties of the power distribution. Although various power distributions functonal forms have been proposed for the univariate marginals at the transmitter and receiver, little has been suggested in terms of bivariate distributions for $\mathcal{P}(\theta, \phi)$. We propose natural extensions of the univariate marginals, the uniform limited, Gaussian and Laplacian distributions [6], to the bi-variate case. These distributions are parametrized by a mean angle-of-arrival $\theta_{0}$, mean angle-of-departure $\phi_{0}$, angular spread at the transmitter $\sigma_{t}$ and receiver $\sigma_{r}$, and parameter $\rho$ controlling joint properties. They are illustrated in Fig. 2.

\section{A. Morgenstern Distributed Scatterers}

In the case the energy leaves the transmitter aperture uniformly from $\left(\theta_{0}-\Delta_{t}, \theta_{0}+\Delta_{t}\right)$ to arrive at the receiver aperture uniformly from $\left(\theta_{0}-\Delta_{r}, \theta_{0}+\Delta_{r}\right)$, marginal constraints (4) can be satisfied by Morgenstern's family of distributions [9]:

$$
f_{\mathrm{M}}(\theta, \phi)=\frac{1}{4 \Delta_{t} \Delta_{r}}-\frac{\rho_{m}\left(\theta-\theta_{0}\right)\left(\phi-\phi_{0}\right)}{4 \Delta_{t}^{2} \Delta_{r}^{2}} .
$$

Using (5b) it is straight-forward to derive:

$$
\gamma_{\ell \ell^{\prime}}^{(\mathrm{M})}=\left\{\begin{array}{cc}
e^{i \ell \theta_{0}} \operatorname{sinc}\left(\ell \Delta_{t}\right), & \ell^{\prime}=0 \\
e^{-i \ell^{\prime} \phi_{0}} \operatorname{sinc}\left(\ell^{\prime} \Delta_{r}\right), & \ell=0 \\
e^{i\left(\ell \theta_{0}-\ell^{\prime} \theta_{0}\right)} \Gamma\left(\ell, \ell^{\prime}\right), & \text { otherwise }
\end{array}\right.
$$

where

$$
\begin{aligned}
\Gamma\left(\ell, \ell^{\prime}\right)= & \operatorname{sinc}\left(\ell \Delta_{t}\right) \operatorname{sinc}\left(\ell^{\prime} \Delta_{r}\right)-\frac{\rho}{\ell \Delta_{t} \ell^{\prime} \Delta_{r}} \times \\
& {\left[\cos \left(\ell \Delta_{t}\right)-\operatorname{sinc}\left(\ell \Delta_{t}\right)\right]\left[\cos \left(\ell^{\prime} \Delta_{r}\right)-\operatorname{sinc}\left(\ell^{\prime} \Delta_{r}\right)\right] . }
\end{aligned}
$$

Parameter $\rho \in[-1,1]$ controls the shape of $f_{\mathrm{M}}(\theta, \phi)$. In case $\rho=0$, the distribution reduces to the separable product of two uniform limited distributions, the Kronecker case. Further in the isotropic case $\Delta_{t}=\pi$ and $\Delta_{r}=\pi$ the Morgenstern distribution reduces to the rich scattering distribution.

\section{B. Gaussian Distributed Scatterers}

The truncated bivariate Gaussian distribution is written:

$$
f_{\mathrm{G}}(\theta, \phi)=\Omega_{\mathrm{G}} e^{-\frac{Q(\theta, \phi)}{2\left(1-\rho^{2}\right)}},\left|\theta-\theta_{0}\right|,\left|\phi-\phi_{0}\right| \leq \pi
$$

where

$$
Q(\theta, \phi)=\frac{\left(\theta-\theta_{0}\right)^{2}}{\sigma_{t}^{2}}-\frac{2 \rho\left(\theta-\theta_{0}\right)\left(\phi-\phi_{0}\right)}{\sigma_{t} \sigma_{r}}+\frac{\left(\phi-\phi_{0}\right)^{2}}{\sigma_{r}^{2}},
$$

$\rho$ controls the level of coupling between the transmitter and receiver scatterers and $\Omega_{\mathrm{G}}$ is a normalization constant. The Kronecker case is obtained by setting $\rho=0$. For small angular spread $\left(\sigma_{r}, \sigma_{t} \ll \pi\right), \Omega_{\mathrm{G}}=1 / 2 \pi \sqrt{1-\rho^{2}} \sigma_{t} \sigma_{r}$ and coefficients $\gamma_{\ell \ell^{\prime}}$ are obtained from the Gaussian characteristic function [10]:

$$
\gamma_{\ell \ell^{\prime}}^{(\mathrm{G})}=e^{i\left(\ell \theta_{0}-\ell^{\prime} \phi_{0}\right)} e^{-\frac{1}{2}\left[\ell^{2} \sigma_{t}^{2}+\ell^{\prime 2} \sigma_{r}^{2}-2 \sigma_{t} \sigma_{r} \rho \ell \ell^{\prime}\right]}
$$

The marginal distributions possess characteristic functions of form $\gamma_{\ell}^{(\mathrm{G})}=e^{i \ell \theta_{0}} e^{-\frac{1}{2} \ell^{2} \sigma_{t}^{2}}$, which correspond to the univariate 


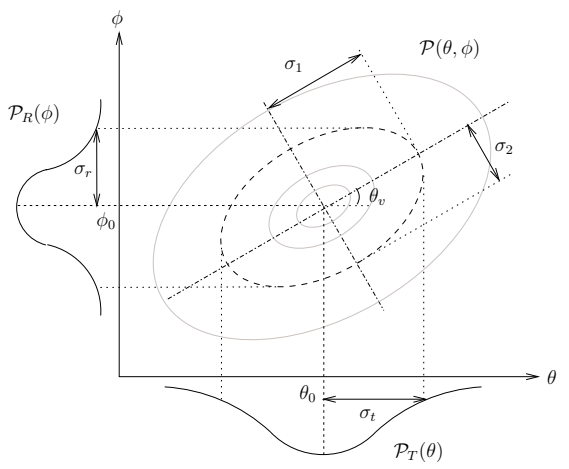

Fig. 3. Contour plot for a typical elliptical bivariate scatterer power distribution, showing distribution parameters: mean direction pair $\left(\theta_{0}, \phi_{0}\right)$ and major and minor axes of ellipses $\sigma_{1}$ and $\sigma_{2}$, respectively. Also shown are the marginal density of scatterers $P_{\mathrm{T}}(\theta)$ and $P_{\mathrm{R}}(\phi)$.

Gaussian distribution $f_{\mathrm{G}}(\theta)=\frac{1}{\sqrt{2 \pi} \sigma_{t}} e^{-\left(\theta-\theta_{0}\right)^{2} / 2 \sigma_{t}^{2}}$ [6]. The variances of the marginals, at the transmitter and receiver, are $\sigma_{t}^{2}$ and $\sigma_{r}^{2}$ respectively.

The distribution is an elliptically contoured function centered about $\left(\theta_{0}, \phi_{0}\right)$ and parameterized in terms the ellipse orientation angle $\theta_{v}$ (see Fig. 3) and major and minor axis variances $\sigma_{1}^{2}$ and $\sigma_{2}^{2}$ respectively. The joint parameter $\rho$ is a function of these geometric parameters [10]:

$$
\rho=\frac{\sigma_{1}^{2}-\sigma_{2}^{2}}{\sigma_{t} \sigma_{r}} \sin \theta_{v} \cos \theta_{v},
$$

where $\sigma_{t}^{2}=\sigma_{1}^{2}+\left(\sigma_{2}^{2}-\sigma_{1}^{2}\right) \sin ^{2} \theta_{v}$ and $\sigma_{r}^{2}=\sigma_{2}^{2}+\left(\sigma_{1}^{2}-\right.$ $\left.\sigma_{2}^{2}\right) \sin ^{2} \theta_{v}$. Geometric parameters are illustrated in Fig. 3 .

\section{Laplacian Distributed Scatterers}

Similarly to the Gaussian distribution, a truncated elliptical bivariate Laplacian distribution can also be written [11]:

$$
f_{\mathrm{L}}(\theta, \phi)=\Omega_{\mathrm{L}} K_{0}\left(\sqrt{\frac{Q(\theta, \phi)}{1-\rho^{2}}}\right),\left|\theta-\theta_{0}\right|,\left|\phi-\phi_{0}\right| \leq \pi,
$$

where $K_{0}(\cdot)$ is the modified Bessel function of the second kind of order zero and $\Omega_{\mathrm{L}}$ is a normalization constant. For small angular spread, $\Omega_{\mathrm{L}}=1 / \pi \sqrt{1-\rho^{2}} \sigma_{t} \sigma_{r}$ and coefficients are obtained from the Laplacian characteristic function [11]:

$$
\gamma_{\ell \ell^{\prime}}^{(\mathrm{L})}=\frac{e^{i\left(\ell \theta_{0}-\ell^{\prime} \phi_{0}\right)}}{1+\sigma_{t}^{2} \ell^{2}+\sigma_{r}^{2} \ell^{\prime 2}-2 \rho \sigma_{t} \sigma_{r} \ell \ell^{\prime}} .
$$

The marginal distributions possess characteristic functions of form $\gamma_{\ell}^{(\mathrm{L})}=e^{i \ell \theta_{0}} /\left(1+\sigma_{t}^{2} \ell^{2}\right)$ which correspond to the well-known univariate Laplacian distribution $f_{\mathrm{L}}(\theta)=$ $\frac{1}{2 \sigma_{t}} e^{-\left|\theta-\theta_{0}\right| / \sigma_{t}}$ with variance $2 \sigma_{t}^{2}$.

\section{Information Theoretic Performance Measures}

We now describe the measures of capacity and diversity used for assessing MIMO system performance. The ergodic capacity of a MIMO channel with no channel state information at the transmitter, is given by computing the mutual information with equally allotted transmit powers [12]:

$$
C_{\text {erg }}=E_{\boldsymbol{H}}\left\{\log _{2}\left|\boldsymbol{I}_{n_{\mathrm{T}}}+\frac{\mathrm{SNR}}{n_{\mathrm{R}}} \boldsymbol{H} \boldsymbol{H}^{\dagger}\right|\right\},
$$

where $\boldsymbol{I}_{n_{\mathrm{T}}}$ is the $n_{\mathrm{T}} \times n_{\mathrm{T}}$ identity matrix and SNR the average received signal-to-noise ratio.

Channel diversity is generally defined by the distribution of the $n_{\mathrm{R}} n_{\mathrm{T}}$ eigenvalues $\lambda_{i}$ of the channel correlation matrix $\boldsymbol{R}_{\boldsymbol{H}}$. These eigenvalues describe the average powers of the independent eigenvector channels of a MIMO channel. A useful single parameter diversity measure describing the eigenvalue spread is the diversity order. In [13], for an NLOS channel with Gaussian coefficients it was shown equal to:

$$
\Psi\left(\boldsymbol{R}_{\boldsymbol{H}}\right)=\frac{\left(\sum_{i=1}^{n_{\mathrm{R}} n_{\mathrm{T}}} \lambda_{i}\right)^{2}}{\sum_{i=1}^{n_{\mathrm{R}} n_{\mathrm{T}}} \lambda_{i}^{2}}=\left(\frac{\operatorname{Tr}\left\{\boldsymbol{R}_{\boldsymbol{H}}\right\}}{\left\|\boldsymbol{R}_{\boldsymbol{H}}\right\|_{F}}\right)^{2},
$$

where $\|\cdot\|_{F}$ is the Frobenius norm and $\operatorname{Tr}(\cdot)$ is the matrix trace. Here $1<\Psi\left(\boldsymbol{R}_{\boldsymbol{H}}\right)<n_{\mathrm{T}} n_{\mathrm{R}}$.

To obtain antenna geometry-independent performance results for our antenna apertures, we set $\boldsymbol{J}_{\mathrm{T}}^{\dagger} \boldsymbol{J}_{\mathrm{T}}=\boldsymbol{I}_{n_{T}}$ and $\boldsymbol{J}_{\mathrm{R}}^{\dagger} \boldsymbol{J}_{\mathrm{R}}=\boldsymbol{I}_{n_{R}}$. These relations are satisfied for $n_{\mathrm{T}} \geq 2 N_{\mathrm{T}}+1$ transmitter and $n_{\mathrm{R}} \geq 2 N_{\mathrm{R}}+1$ receiver antennas that are separated far enough apart so that the rich scattering $\left(\boldsymbol{R}_{\boldsymbol{H}}=\right.$ I) antenna correlation is negligible.

Using (8), we see immediately that:

$$
\boldsymbol{H} \boldsymbol{H}^{\dagger}=\boldsymbol{J}_{\mathrm{R}} \boldsymbol{\beta} \boldsymbol{J}_{\mathrm{T}}^{\dagger} \boldsymbol{J}_{\mathrm{T}} \boldsymbol{\beta}^{\dagger} \boldsymbol{J}_{\mathrm{R}}^{\dagger}=\left(\boldsymbol{J}_{\mathrm{R}} \boldsymbol{\beta}\right)\left(\boldsymbol{\beta}^{\dagger} \boldsymbol{J}_{\mathrm{R}}^{\dagger}\right) .
$$

For fixed antennas, computing expectation (14) over channel realizations $\boldsymbol{H}$ is equivalent to computing it over $g(\theta, \phi)$ or $\boldsymbol{\beta}$. Using the matrix identity $|\boldsymbol{I}+\boldsymbol{A} \boldsymbol{B}|=|\boldsymbol{I}+\boldsymbol{B} \boldsymbol{A}|$ :

$$
\begin{aligned}
C_{\mathrm{erg}} & =E_{\boldsymbol{\beta}}\left\{\log _{2}\left|\boldsymbol{I}_{n_{\mathrm{T}}}+\frac{\mathrm{SNR}}{n_{\mathrm{R}}} \boldsymbol{\beta}^{\dagger} \boldsymbol{J}_{\mathrm{R}}^{\dagger} \boldsymbol{J}_{\mathrm{R}} \boldsymbol{\beta}\right|\right\} \\
& =E_{\boldsymbol{\beta}}\left\{\log _{2}\left|\boldsymbol{I}_{n_{\mathrm{T}}}+\frac{\mathrm{SNR}}{n_{\mathrm{R}}} \boldsymbol{\beta}^{\dagger} \boldsymbol{\beta}\right|\right\} .
\end{aligned}
$$

Similarly for the diversity, using Kronecker product property $(\boldsymbol{A} \otimes \boldsymbol{B})(\boldsymbol{C} \otimes D)=\boldsymbol{A C} \otimes \boldsymbol{B D}:$

$$
\left(\boldsymbol{J}_{\mathrm{T}}^{*} \otimes \boldsymbol{J}_{\mathrm{R}}\right)^{\dagger}\left(\boldsymbol{J}_{\mathrm{T}}^{*} \otimes \boldsymbol{J}_{\mathrm{R}}\right)=\boldsymbol{J}_{\mathrm{T}}^{T} \boldsymbol{J}_{\mathrm{T}}^{*} \otimes \boldsymbol{J}_{\mathrm{R}}^{\dagger} \boldsymbol{J}_{\mathrm{R}}=\boldsymbol{I}_{n_{T} n_{R}}
$$

Hence from (13), $\boldsymbol{R}_{\boldsymbol{H}}$ and $\boldsymbol{R}_{\boldsymbol{\beta}}$ are similar matrices and possess the same trace and Frobenius norm. As a result, diversity order is given by:

$$
\Psi\left(\boldsymbol{R}_{\boldsymbol{H}}\right)=\left(\frac{\operatorname{Tr}\left\{\boldsymbol{R}_{\boldsymbol{\beta}}\right\}}{\left\|\boldsymbol{R}_{\boldsymbol{\beta}}\right\|_{F}}\right)^{2} .
$$

From (16) and (17), both capacity and diversity are hence characterized by the scattering matrix $\boldsymbol{\beta}$.

\section{Simulation of Jointly Correlated Channels}

In the following examples, we use the above metrics to investigate the joint properties of the MIMO channel. Here antennas are packed into apertures of radius $R_{\mathrm{T}}=R_{\mathrm{R}}=\lambda$.

Ergodic capacity $C_{\text {erg }}$ was calculated by ensemble averaging over 1000 realizations of the scatter matrix $\boldsymbol{\beta}$. SNR was 
(a)

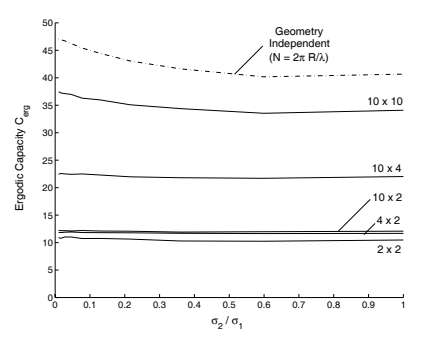

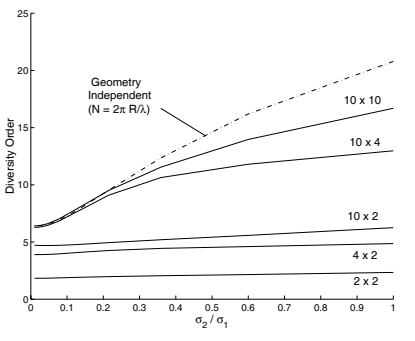

(b)
Fig. 4. (a) Ergodic capacity and (b) diversity order for various MIMO antenna schemes in a scattering environment with bivariate Gaussian angular power Here $\theta_{v}=45^{\circ}, \theta_{t}=\theta_{r}=0.5$ while the major-minor axis ratio $\alpha$ is varied. Shown are geometry independent measures (dotted lines).

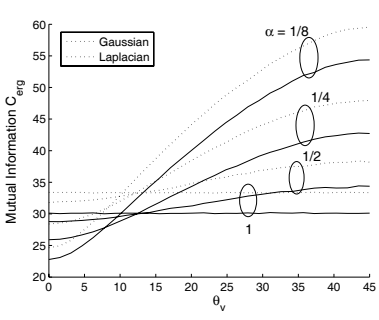

(a)

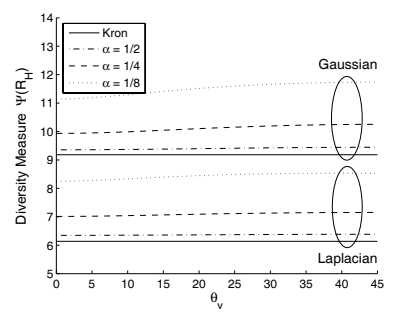

(b)
Fig. 5. (a) Ergodic capacity and (b) diversity order for Gaussian (Laplacian) distibution when varying the orientation angle $\theta_{v}$, for constant ellipse area $\pi \sigma_{1} \sigma_{2}=0.1 \pi(0.2 \pi)$ but different major-minor axis ratio $\alpha$.

set to $20 \mathrm{~dB}$. The set of channel scatter matrices $\left\{\boldsymbol{\beta}_{n}\right\}$ was simulated from the coefficient correlation matrix $\boldsymbol{R}_{\beta}$ by: (i) generating a series of $\left(2 N_{\mathrm{T}}+1\right)\left(2 N_{\mathrm{R}}+1\right)$-length vectors of IID zero mean unit variance circularly symmetric Gaussian random variables $\left\{\overrightarrow{\boldsymbol{G}}_{i}\right\}$, and (ii) calculating realizations of the scatter matrix using $\overrightarrow{\boldsymbol{\beta}}_{i}=\boldsymbol{R}_{\boldsymbol{\beta}}{ }^{1 / 2} \overrightarrow{\boldsymbol{G}}_{i}$.

\section{A. Jointness of Power Distributions}

We first study the effect of keeping the scatterer densities around the transmitter and receiver constant, while varying the joint parameter through the elliptical distribution parameters: ellipse major-axis-to-minor-axis ratio $\alpha \triangleq \sigma_{2} / \sigma_{1}$ and elliptical contour orientation $\theta_{v}$.

Anticipating largest joint effects occur for ellipses oriented at $\theta_{v}=45^{\circ}$, we plot the measures against $\alpha$ in Fig. 4. Here maximum distribution jointness $(\alpha=0)$ offers a modest $15 \%$ boost to capacity but a large drop in diversity. Though plotted for Gaussian scatterers with $\theta_{t}=\theta_{r}=0.5$, sameshaped curves result for other angular spreads and Laplacian scatterers.

Fig. 4 also compares the geometry independent performance measures with those calculated directly from (14) and (15), for antenna configured into uniform circular arrays of radius $\lambda$. The geometry independent measures are reasonably close to the $10 \times 10$ results.

\section{B. Orientation of the Distribution Contours}

We also study the effect of orientation of the distribution contours, holding the contour area $\pi \sigma_{1} \sigma_{2}$ of the power dis- tribution constant. In Fig. 5, we see capacity depends significantly on contour orientation, while diversity order is relatively fixed. Further simulations show that diversity order is even less strongly dependent upon orientation for smaller aperture sizes. Diversity order shows much stronger dependence upon contour area (which depends linearly on $\alpha$ ) than contour orientation. However, contour orientation is shown important for modeling MIMO channel capacity.

\section{CONCLUSION}

In this paper, we explore the impact of the joint properties of the angular power distribution on MIMO system performance. We present a novel NLOS modelling approach to generate an antenna geometry-free analysis of the MIMO channel. In doing so, we show that the joint properties can significantly effect diversity order and ergodic capacity. Capacity is strongly dependent upon distribution contour orientation while the diversity order proportional to the area of distribution contours.

\section{ACKNOWLEDGMENT}

This work was partially funded by Australian Research Council Discovery Grant number DP0343804.

\section{REFERENCES}

[1] K. Yu, M. Bengtsson, B. Ottersten, D. McNamara, P. Karlsson, and M. Beach, "Second order statistics of NLOS indoor MIMO channels based on $5.2 \mathrm{GHz}$ measurements," in IEEE Global Communications Conf., Nov. 2001.

[2] Q. H. Spencer, B. D. Jeffs, M. A. Jensen, and A. L. Swindlehurst, "Modeling the statistical time and angle of arrival characeristics of an indoor multipath channel," IEEE J. Select. Areas Commun., vol. 18, no. 3, pp. $347-360,2000$.

[3] H. Ozcelik, N. Czink, and E. Bonek, "What makes a good MIMO channel model," in Vehicular Technology Conference, vol. I, pp. 156 $-160,2005$.

[4] T. S. Pollock, "Correlation modelliing in MIMO systems: when can we Kronecker?," in Proc. 5th Australian Communications Theory Workshop, pp. 149 - 153, 2004.

[5] P. A. Bello, "Characterization of randomly time-variant linear channels," IEEE Trans. Comm. Sys., vol. 11, pp. 360 - 393, 1963.

[6] P. Teal and T. A. Abhayapala, "Spatial correlation in non-isotropic scattering scenarios," in Proc. IEEE International Conference on Acoustics, Speech and Signal Processing, vol. III, pp. 2833 - 2866, 2002.

[7] T. Abhayapala, T. Pollock, and R. Kennedy, "Spatial decomposition of MIMO wireless channels," in Proc. IEEE Seventh International Symposium on Signal Processing and its Applications, ISSPA 2003, vol. 1, pp. 309-312, July 2003.

[8] A. Graham, Kronecker products and matrix calculus with applications. Chichester: Ellis Horwood, 1981.

[9] N. L. Johnson and S. Kotz, "On some generalized Farlie-GumbelMorgenstein distribution," Communications in Statistics, vol. 4, pp. 415 - 427, 1975.

[10] T. Betlehem, T. D. Abhayapala, and T. A. Lamahewa, "Space-time MIMO channel modelling using angular power distributions," in Proc. 7th Australian Communications Theory Workshop, (to appear), 2006.

[11] T. J. Kozubowski and K. Podgorski, "A multivariate and asymmetric generalization of Laplace distribution," Computational Statistics, vol. 15 , no. 4, pp. $531-540,2000$.

[12] G. J. Foschini and M. J. Gans, "On limits of wireless communications in fading environments when using multiple antennas," Wireless Personal Communications, vol. 6, pp. 311 - 335, 1998.

[13] M. T. Ivrlac and J. A. Nossek, "Quantifying diversity and correlation of Rayleigh fading MIMO channels," in IEEE International Symposium on Signal Processing and Information Technology, ISSPIT'03, 2003. 